\title{
Effectiveness of Hope Therapy Protocol on Depression and Hope in Amphetamine Users
}

\author{
Hasan Sadeghi, ${ }^{1, *}$ Leyla Ebrahimi, ${ }^{2}$ and Leyla Vatandoust ${ }^{3}$ \\ ${ }^{1}$ Department of Psychology, Young Researchers and Elite Club, Ardabil Branch, Islamic Azad University, Ardabil, IR Iran \\ ${ }^{2}$ Department of Psychology, University of Allameh Tabataba'i, Tehran, IR Iran \\ ${ }^{3}$ Young Researchers and Elite Club, Sari Branch, Islamic Azad University, Sari, IR Iran \\ ${ }^{*}$ Corresponding author: Hasan Sadeghi, Department of Psychology, Young Researchers and Elite Club, Ardabil Branch, Islamic Azad University, Ardabil, IR Iran. Tel: +98-9122499434, \\ E-mail:sadeghi_66@yahoo.com
}

Received 2014 July 9; Revised 2014 December 14; Accepted 2015 February 18.

\begin{abstract}
Background: Addiction has surpassed the boundaries of health and treatment and turned into a social crisis and a debilitating and major concern in today's world. Amphetamine, one of the addictive drugs, is classified as psycho-stimulants drugs, which increase arousal, alertness, and motor activity. Humans report that this drug produces a significant euphoria and is highly addictive.

Objectives: The present study aimed to evaluate the effectiveness of hope therapy protocol (HTP) on depression reduction and hope increase in amphetamine users.

Patients and Methods: This study has a quasi-experimental design with experimental and control groups. The sample included all amphetamine consumers referring to day drug addiction treatment center in Ray City, Iran, selected with convenience method. In order to analyze the data, multivariate analysis of covariance (MANCOVA) was applied using SPSS software.

Results: The results showed that $\mathrm{F}$ value of mean scores in depression and hope post-tests of the experimental and control groups are 24.94 and 25.73 , respectively, which are significant $(\mathrm{P}<0.01)$. Therefore, hope therapy training could reduce depressive symptoms in amphetamine consumers and improve their hope.

Conclusions: Performing HTP can improve hopefulness and symptoms of patients, specially addicted ones. In addition, it can prevent substance abusers from returning to drugs and leaving the treatment period unfinished.
\end{abstract}

Keywords: Therapy, Depression, Hope, Amphetamine

\section{Background}

Today, addiction problem has gone beyond the boundaries of health and treatment. It has turned into a social crisis and a debilitating and major concern in today's world. Since drug dependence has inhibitory effects on the growth and prosperity of the community, it should be attended as a serious threat and one of the most general health problems all over the world (1).

Drug addiction is a painful reality, especially for adolescents and young adults. It is one of the most important social, economic, and health problems with serious threats for human societies which may result in social stagnation in various fields (2). In addition, creation of synthetic and laboratory materials has increased difficulties of coping with this problem. One of these unsafe substances is metamphetamine. Amphetamine and metamphetamine have similar mechanism in the body and both release monoamine transmitter, i.e. dopamine, serotonin, and norepinephrine (3).

Using amphetamines creates an explosion of energy in the consumer. They increase activity, reduce appe- tite and overall, make a pleasing feeling like euphoria. Therefore, when a person takes these drugs, he or she does not eat, sleep, or rest since he or she has no appetite to eat and does not feel tired or need to sleep $(1,4)$. Epidemiologic information shows that substance abuse in depressed individuals is significantly higher than others $(5,6)$. However, it is unclear whether substance abuse increase depression or vice versa $(3,6)$. Prevalence of depression in drug users, depending on the kind of population and return to use, is between $16 \%$ and $44 \%$ (7, 8). According to clinical observations, substance abuse disorders are often correlated with some mental, emotional, behavioral, and mood disorders such as depression and anxiety.

Depression is the feeling of gloom and sorrow that lasts for at least 2 weeks and is different from mental stress pressure or feeling sad for losing something. Despair is one of the main characteristics of depression. If you ask people diagnosed with major depressive disorder what is the worst thing in their lives, they refer to their depres-

Copyright (C) 2015, Zahedan University of Medical Sciences. This is an open-access article distributed under the terms of the Creative Commons Attribution-NonCommercial 4.0 International License (http://creativecommons.org/licenses/by-nc/4.0/) which permits copy and redistribute the material just in noncommercial usages, provided the original work is properly cited. 
sion. They also believe that their depression will continue and never be treated (9).

Cheavens et al. (10) argue that these individuals are depressed about their depressive states. Depression during drug dependence has consequences in terms of mortality and comorbidity with other disorders that are far more important for the people than their depression. People suffering from depression, do not response appropriately to antiviral treatments (11), are at greater risk of taking high dose drugs (8) and committing suicide (12), as well as higher rate of return to drug abuse (13). In addition, previous studies show that depression and anxiety have been less reported in hopeful individuals and low hope can be the predictor of depression level and mental/social weaknesses that are among the main factors of a qualified life (14).

Hope, as a fundamental personal characteristic and source of energy in people's lives, is a healing and enhancing welfare factor (15). Snyder and Rand (14) also believed that hopeful people response more actively when confronting stressful situations and use more and better coping strategies. From this theoretical perspective, hopelessness results in developing or exacerbating depression and threats mental health, which in turn, increases physical problems. Hope, as an essential element for effective treatment of mental disorders, has a healing impact (15). Although hopefulness levels vary among people, it is a relatively stable character (16).

The most research study in hope therapy protocol (HTP) in recent decades belongs to Seligman (17), the father of positive psychology, Snyder and Rand (14), and Snyder et al. (18). Their studies clarified that hopelessness can develop various disorders and those with substance abuse are more in danger of returning to drug abuse in disappointing situations $(9,14)$.

HTP tries to help clients to set clear goals, categorize them, and consider various ways to attain them. It also motivates them to pursue their goals and turns obstacles into challenges $(2,14,15)$. In a study, Cheavens et al. (2) showed that performing HTP could reduce basic depression symptoms in depressed individuals. In another study, performing HTP on patients with psychological diseases resulted in relative improvement (19). Also, based on the research of Raisian et al. (20) training package of HTP could reduce depression symptoms in substance abusers. Therefore, given the undeniable effect of HTP in improvement of mental disorders, its positive effects in prevention of return to substance abuse, as well as lack of studies on the effect of this therapy on metamphetamine consumers, we decided to investigate the effectiveness of HTP on depression reduction and hope increase in metamphetamine consumers. The main question is that whether performing HTP on depressed metamphetamine users can reduce depression symptoms and increase hope in them.

\section{Objectives}

The present study aimed to evaluate the effectiveness of
HTP on depression reduction and hope increase in amphetamine users.

\section{Patients and Methods}

Design of the present study was quasi-experimental with a control group and taking pretest and posttest. The statistical population consisted of all amphetamine drug consumers referring to day drug addiction treatment center in Rey City, Iran. The following instruments and protocol were used to assess and train the participants.

\subsection{Beck Depression Inventory}

This test was designed by Beck in 1961 (21). It was revised in 1971 and published in 1978. Beck depression inventory consisted of 21 items related to different symptoms. The subject is asked to rate the symptoms in a 0 to 3 measurement scale. The correlation of this inventory is 0.71 with Hamilton depression rating scale and its test-retest reliability after one week was calculated as 0.93. Its internal consistency is also 0.91 (22). Bakhshani (23) reported its $\alpha$ coefficient as 0.91 , correlation coefficient as 0.89 , and test-retest coefficient as 0.94 .

\subsection{Snyder Hope Scale}

This scale, introduced by Schneider and colleagues in 1991, is made of two subscales of crossing (identifying different crosses, paths, and ways to reach a goal) and agent (decision to reach the goal). It takes 2 to 5 minutes to do this scale. Each question is answered on a 1 to 4 rating measurement from "totally false" to "totally true". The questions $3,5,7$, and 11 are distractions and are not scored. Former studies have confirmed its internal reliability (18). In addition, Gulzar (24) reported its reliability with internal consistency and the Cronbach $\alpha$ coefficient as 0.89 .

\subsection{Hope Therapy Protocol}

It includes defining hope and its various aspects such as introducing goals and its kinds as a part of hope increase process, making a framework for discovering goals, presenting strategies to set clear and realistic goals in various levels, preparing a list of goals, prioritizing them and selecting one goal to be worked at in the group, highlighting the importance of intention, goal, and meaning in the life, presenting ways of increasing motivation and mental and physical will in pursuing goals, and improving the role of hope in depression symptoms and goals persuasion (18). The reliability for hope therapy reported 0.76 . Cheavens et al. (25) study has indicated acceptable internal consistency for the State Hope Therapy with alphas ranging from 0.79 to 0.95 .

\subsection{Procedure}

After coordination with the center management, 2 volunteer groups were formed with 25 members in each, 
from metamphetamine consumers with depression symptoms (confirmed diagnosis of depression disorder). To evaluate the effectiveness of the intervention, a pretest was taken from both groups. Then, in 8 sessions, HTP was performed on the experimental group and at the end of the intervention, all subjects participated in the posttest. Inclusion criteria of research were as follows: use of amphetamines, diagnosis of depression, willingness to participate, and being 18 to 25 years old. Also exclusion criteria of research were twice absence from training HTP sessions and use of other psychoactive substances or drugs during the course. In the present study, the experimental group (amphetamines consumers with depressive symptoms) was under the supervision of a psychiatrist and did not take antidepressants. The HTP started 1 month after discontinuation of Amphetamine use.

\section{Results}

About 70\% of the participants were between 18 and 24 years old and the rest were between 25 and 28 years old. Also, $60 \%$ of participants had a diploma and post diploma and the $40 \%$ of them had a graduate and higher degree.

As it can be seen in Table 1, the highest standard deviation and mean belong to "education level" and "age" of the control group, respectively.

According to Table 2, the highest standard deviation and mean belong to depression in the experimental and hope in the control groups, respectively.

The results of Box Test show that Box Test is not significant (Box $=42.92)$, i.e. the differences between covariance are not significant. According to Table 3 there was a significant relation between pretest scores of both depression and hope variables and their posttest scores $(\mathrm{P}<0.01)$.

According to Table 4, the group effect on combination of posttest scores was significant. In other words, there was a significant relation between the experimental and control groups at least in one of the depression and hope variables. Eta value showed that 0.48 of combinational variable variance was determined by group membership of the subjects.

As seen in Table 5, F of mean scores in depression and hope posttests of the experimental and control groups are 24.94 and 25.73, respectively, which are significant ( $P$ $<0.01$ ). Based on Eta (effect) coefficient, the differences between depression and hope variables in 2 groups were related to the influence of teaching HTP.

\begin{tabular}{lc}
\hline Table 1. Mean and Standard Deviation of the 2 Groups $^{\mathrm{a}}$ \\
\hline Group & Mean \pm Standard Deviation \\
\hline Experimental & $1.86 \pm 0.50$ \\
Age & $1.36 \pm 0.49$ \\
Education level & \\
Control & $2.12 \pm 0.60$ \\
Age & $1.72 \pm 0.61$ \\
\hline Education level
\end{tabular}

$\mathrm{a}_{\mathrm{N}=25}$.

Table 2. Mean and Standard Deviation of Depression and Hope ${ }^{\mathrm{a}}$

\begin{tabular}{lc}
\hline Group & Mean \pm Standard Deviation \\
\hline Depression & \\
Age & $16.36 \pm 2.88$ \\
Education level & $16.80 \pm 2.53$ \\
Hope & \\
Age & $20.27 \pm 1.51$ \\
Education level & $23.56 \pm 1.57$ \\
\hline $\mathrm{a}_{\mathrm{N}}=25$ & \\
\hline
\end{tabular}

Table 3. Results of MANCOVA Analysis on Variables of Depression and Hope

\begin{tabular}{lllcll}
\hline Test Name & Value & F & Hypothesis df & Error df & P Value \\
\hline Depression & & & & 38 & 0.000 \\
\hline Pilayi effect & 0.076 & 0.78 & 4 & 38 & 0.000 \\
\hline Wilks' lambda & 0.92 & 0.78 & 4 & 38 & 0.000 \\
\hline Hotelling effect & 0.08 & 0.78 & 4 & 38 & 0.000 \\
\hline Largest root & 0.08 & 0.78 & 4 & 38 & 0.000 \\
\hline Hope & & & 4 & 0.000 \\
\hline Pilayi Effect & 0.44 & 7.30 & 4 & 38 & 0.000 \\
\hline Wilks' lambda & 0.57 & 7.30 & 4 & 38 & 0.000 \\
\hline Hotelling effect & 0.77 & 7.30 & 4 & 48 \\
\hline Largest root & 0.77 & 7.30 & 4 & & \\
\hline
\end{tabular}


Sadeghi Het al.

\begin{tabular}{lcccccc}
\hline Table 4. Results of MANCOVA Analysis in the Group & & & & & \\
\hline Depression Test Name & Value & F & Hypothesis df & Error df & P Value & Eta \\
\hline Pilayi effect & 0.49 & 9.07 & 4 & 38 & 0.000 & 0.498 \\
Willks' lambda & 0.51 & 9.07 & 4 & 38 & 0.000 & 0.498 \\
Hotelling effect & 0.96 & 9.07 & 4 & 38 & 0.000 & 0.498 \\
Largest root & 0.96 & 9.07 & 4 & 38 & 0.000 & 0.498 \\
\hline
\end{tabular}

Table 5. Results of Hope Therapy Protocol Training on Improvement of Depression and Hope

\begin{tabular}{lcccccc}
\hline Group & SS & df & MS & F & P Value & Eta Square \\
\hline Depression & 54.12 & 1 & 54.12 & 24.94 & 0.000 & 0.37 \\
Hope & 67.25 & 1 & 67.25 & 25.73 & 0.000 & 0.386 \\
\hline
\end{tabular}

\section{Discussion}

The aim of the present study was to investigate the effectiveness of HTP on depression reduction and hope increase in metamphetamine users. The results revealed that this protocol was successful in reducing depression symptoms in them. This finding is consistent with those of previous studies $(15,18)$. Kao et al. $(26)$ demonstrated that positive psychological approaches are effective in reducing symptoms of various disorders, including anxiety, OCD, depression, or even psychotic disorders.

One obvious characteristic of people suffering from depression is the loss of hope and motivation to challenge problems. This lack creates difficulties for them in confronting problems in social, occupational, familial, and personal situations (15). Therefore, HTP helps depressed individuals realize their status because it establishes a framework for discovering goals, presents strategies to set clear and realistic goals in different levels, and aids to prepare a list of goals and prioritizing them. This recognition returns the patient from astray course in such a way that they can reach self-regulation level and participate in the treatment process $(2,27)$. Also, Kortte et al. (28) have introduced positive psychology components (hope, forgiveness, etc.) as predictors of mental welfare and reduction of mental disorders. HTP teaches individuals to think about things still possess and are valuable for them, because patients, especially depressed ones, intentionally think that there is no worthy thing to live for and this defective feeling worsen their learned hopelessness $(9,17)$.

The results of the present study also revealed that HTP could increase hope in metamphetamine users. This finding is consistent with findings of previous studies $(14,18$, 19). Cheavens and Stigen (27) investigated the effectiveness of HTP on hope increase in substance abusers and observed that this approach was effective. In addition, these researchers in their study entitled "hope theory and HTP" introduced hope as the resistance source of individuals against problems and declared that teaching hopefulness can have positive impacts on both healthy and unhealthy persons. That is why many studies consider hope as a resource of resistance that can have positive effects on health and mental welfare while confronting a stressful situation (28).

\section{Acknowledgments}

The authors would like to thanks all people who contributed in this project.

\section{Footnotes}

Authors' Contribution:Hasan Sadeghi conceived and designed the study, performed the statistical analysis, and drafted the manuscript; Leyla Ebrahimi and Leyla Vatandoust reviewed the study and collected the clinical data.

Funding/Support:The study was funded by Young Researchers and Elite Club, Ardabil Branch, Islamic Azad University, Ardabil, Iran.

\section{References}

1. Bulin SE, Masiulis I, Rivera PD, Eisch AJ. Chapter 30 - Addiction, Hippocampal Neurogenesis, and Neuroplasticity in the Adult Brain. In: Miller PM, editor. Biological Research on Addiction. San Diego: Academic Press; 2013. pp. 291-303.

2. Cheavens JS, Feldman DB, Gum A, Michael ST, Snyder CR. Hope therapy in a community sample: A pilot investigation. Soc Indic Res. 2006;77(1):61-78. doi: 10.1007/s11205-005-5553-0.

3. Fergusson DM, Boden JM, Horwood LJ. Tests of causal links between alcohol abuse or dependence and major depression. Arch Gen Psychiatry. 2009;66(3):260-6. doi: 10.1001/archgenpsychiatry.2008.543. [PubMed:19255375]

4. Knackstedt LA. Chapter 56 - Neuropharmacology of Cocaine and Amphetamine. In: Miller PM, editor. Biological Research on Addiction. San Diego: Academic Press; 2013. pp. 573-7.

5. Davis L, Uezato A, Newell JM, Frazier E. Major depression and comorbid substance use disorders. Curr Opin Psychiatry. 2008;21(1):14-8. doi: 10.1097/YCO.0b013e3282f32408. [PubMed: 18281835]

6. Renoir T, Pang TY, Lanfumey L. Drug withdrawal-induced depression: serotonergic and plasticity changes in animal models. Neurosci Biobehav Rev. 2012;36(1):696-726. doi: 10.1016/j.neubio- 
rev.2011.10.003. [PubMed: 22037449]

7. Havard A, Teesson M, Darke S, Ross J. Depression among heroin users: 12-Month outcomes from the Australian Treatment Outcome Study (ATOS). J Subst Abuse Treat. 2006;30(4):355-62. doi: 10.1016/j.jsat.2006.03.012. [PubMed:16716851]

8. Sordo L, Chahua M, Bravo MJ, Barrio G, Brugal MT, DomingoSalvany A, et al. Depression among regular heroin users: the influence of gender. Addict Behav. 2012;37(1):148-52. doi: 10.1016/j. addbeh.2011.09.009. [PubMed: 21968230]

9. Bolier L, Haverman M, Westerhof GJ, Riper H, Smit F, Bohlmeijer E. Positive psychology interventions: a meta-analysis of randomized controlled studies. BMC Public Health. 2013;13:119. doi: 10.1186/1471-2458-13-119. [PubMed: 23390882]

10. Cheavens JS, Feldman DB, Woodward JT, Snyder CR. Hope in cognitive psychotherapies: On working with client strengths. $J$ cognit Psychother. 2006;20(2):135-45. doi:10.1891/jcop.20.2.135.

11. Cook JA, Grey DD, Burke-Miller JK, Cohen MH, Vlahov D, Kapadia F, et al. Illicit drug use, depression and their association with highly active antiretroviral therapy in HIV-positive women. Drug Alcohol Depend. 2007;89(1):74-81. doi: 10.1016/j.drugalcdep.2006.12.002. [PubMed: 17291696]

12. Darke S, Mills K, Teesson M, Ross J, Williamson A, Havard A. Patterns of major depression and drug-related problems amongst heroin users across 36 months. Psychiatry Res. 2009;166(1):7-14. doi:10.1016/j.psychres.2007.12.007. [PubMed:19215987]

13. Torrens M, Gilchrist G, Domingo-Salvany A. Psychiatric comorbidity in illicit drug users: substance-induced versus independent disorders. Drug Alcohol Depend. 2011;113(2-3):147-56. doi: 10.1016/j.drugalcdep.2010.07.013. [PubMed: 20801586]

14. Snyder CR, Rand KL. Hopelessness and health. Thousand Oaks, CA: Sage; 2005.

15. Werner S. Subjective well-being, hope, and needs of individuals with serious mental illness. Psychiatry Res. 2012;196(2-3):214-9. doi:10.1016/j.psychres.2011.10.012. [PubMed: 22382051]

16. Schrank B, Stanghellini G, Slade M. Hope in psychiatry: a review of the literature. Acta Psychiatr Scand. 2008;118(6):421-33. doi: 10.1111/j.1600-0447.2008.01271.x. [PubMed: 18851720]

17. Seligman ME, Csikszentmihalyi M. Positive psychology. An introduction. Am Psychol. 2000;55(1):5-14. [PubMed: 11392865]
18. Snyder CR, Ilardi SS, Cheavens JS, Michael ST, Yamhure L, Sympson $\mathrm{S}$. The role of hope in cognitive-behavior therapies. Cognit Ther and Res. 2000;24(6):747-62.

19. Thorup A, Petersen L, Jeppesen P, Nordentoft M. The quality of life among first-episode psychotic patients in the OPUS trial. Schizophr Res. 2010;116(1):27-34. doi:10.1016/j.schres.2009.10.006. [PubMed:19897341]

20. Raisian A, Golzari M, Borjali A. Effectiveness of Hope Therapy On reduction of depression and relapse prevention in women clients with drug-dependent. Q Addict of Addiction Stud and Subt Abuse. 2010;5(17):32-41.

21. Siegert RJ, Tennant A, Turner-Stokes L. Rasch analysis of the Beck Depression Inventory-II in a neurological rehabilitation sample. Disabil Rehabil. 2010;32(1):8-17. doi: 10.3109/09638280902971398. [PubMed:19925272]

22. Zemestani Yamchi M. impact of group supportive psychotherapy intervention with IPT approach in reducing depressive symptoms in addict male patients. Tehran: Allameh Tabatabaei University of Tehran;2007.

23. Bakhshani B. Personality investigates the dimensions of with stressful events and social support in depressed subjects tested cognitive model of depression. Tehran: Tehran University of Medical Sciences; 1999.

24. Gulzar M. Validation Snyder Hope Scale. Allameh Tabatabai University of Tehran. 2006; Not published.

25. Cheavens JS, Feldman DB, Gum A, Michel S, Snyder CR. Hope Therapy in a Community Sample: A Pilot. Social Indicators Research. 2006;77(1):61-78.

26. Kao YC, Liu YP, Chou MK, Cheng TH. Subjective quality of life in patients with chronic schizophrenia: relationships between psychosocial and clinical characteristics. Compr Psychiatry. 2011;52(2):171-80. doi: 10.1016/j.comppsych. 2010.05.008. [PubMed: 21295224]

27. Cheavens JS, Stigen CL. Hope Theory and Hope Therapy. Encyclopedia of the Sciences of Learning.. Berlin, Heidelberg: Springer; 2012. pp. 1446-8.

28. Kortte KB, Gilbert M, Gorman P, Wegener ST. Positive psychological variables in the prediction of life satisfaction after spinal cord injury. Rehabil Psychol. 2010;55(1):40-7. doi: 10.1037/a0018624. [PubMed: 20175633] 